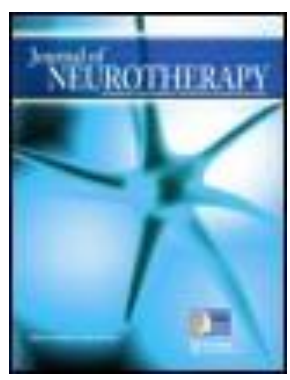

Journal of Neurotherapy: Investigations in Neuromodulation, Neurofeedback and Applied Neuroscience

\title{
When to Inhibit EEG Activity Instead of Reinforcing and Inhibiting Simultaneously
}

\author{
Margaret E. Ayers MA ${ }^{a}$, Marvin W. Sams ND, M. Barry Sterman PhD ${ }^{b}$ \& Joel \\ Lubar PhD ${ }^{\mathrm{C}}$ \\ ${ }^{a}$ Neuropathways EEG Imaging, Inc. , Beverly Hills, California, USA \\ b Sterman-Kaiser Imaging Laboratory, Los Angeles, California, USA \\ C University of Tennessee, Knoxville, Tennessee, USA \\ Published online: 17 Oct 2008.
}

To cite this article: Margaret E. Ayers MA, Marvin W. Sams ND , M. Barry Sterman PhD \& Joel Lubar PhD (2000) WHEN TO INHIBIT EEG ACTIVITY INSTEAD OF REINFORCING AND INHIBITING SIMULTANEOUSLY, Journal of Neurotherapy: Investigations in Neuromodulation, Neurofeedback and Applied Neuroscience, 4:1, 83-93, DOI: $\underline{10.1300 / J 184 v 04 n 01 \_10}$

To link to this article: http://dx.doi.org/10.1300/J184v04n01_10

\section{PLEASE SCROLL DOWN FOR ARTICLE}

(c) International Society for Neurofeedback and Research (ISNR), all rights reserved. This article (the "Article") may be accessed online from ISNR at no charge. The Article may be viewed online, stored in electronic or physical form, or archived for research, teaching, and private study purposes. The Article may be archived in public libraries or university libraries at the direction of said public library or university library. Any other reproduction of the Article for redistribution, sale, resale, loan, sublicensing, systematic supply, or other distribution, including both physical and electronic reproduction for such purposes, is expressly forbidden. Preparing or reproducing derivative works of this article is expressly forbidden. ISNR makes no representation or warranty as to the accuracy or completeness of any content in the Article. From 1995 to 2013 the Journal of Neurotherapy was the official publication of ISNR (www. Isnr.org); on April 27, 2016 ISNR acquired the journal from Taylor \& Francis Group, LLC. In 2014, ISNR established its official open-access journal NeuroRegulation (ISSN: 2373-0587; www. neuroregulation.org).

\section{THIS OPEN-ACCESS CONTENT MADE POSSIBLE BY THESE GENEROUS SPONSORS}

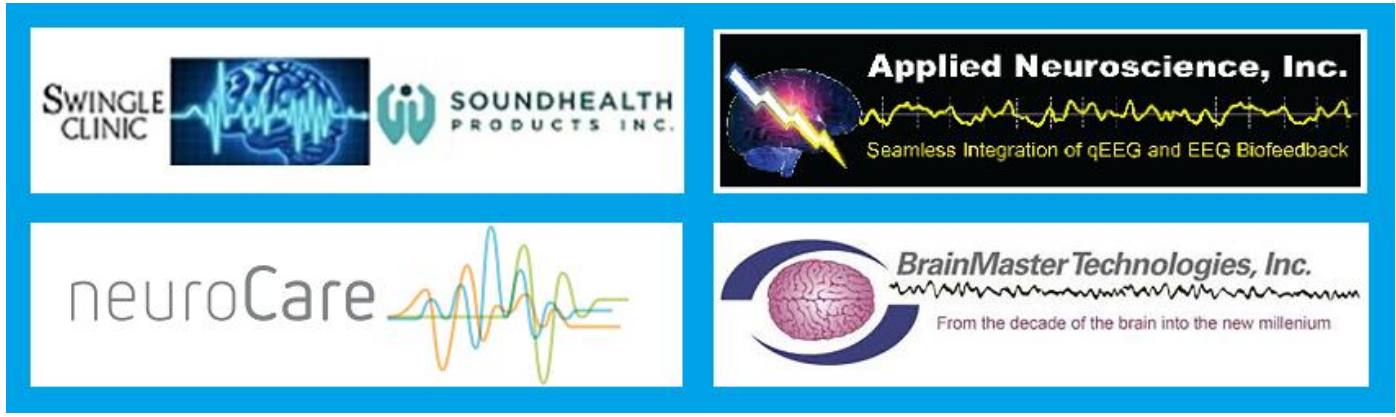




\title{
CLINICAL CORNER
}

\author{
D. Corydon Hammond, PhD, Editor
}

With this issue of the journal, we are beginning a new feature called the Clinical Corner. In it we will pose questions of practical clinical significance to different experienced practitioners in the field to obtain their varying perspectives. You are invited to send questions to the editor of the Clinical Corner section: D. Corydon Hammond, PhD, University Medical Center, PM\&R, Salt Lake City, UT 84132 (E-mail: D.C.Hammond@m.cc.utah.edu).

\section{WHEN TO INHIBIT EEG ACTIVITY INSTEAD OF REINFORCING AND INHIBITING SIMULTANEOUSLY}

QUESTION 1: In neurofeedback, we often talk about training beta and SMR while inhibiting various frequencies, such as theta. What is your opinion about the value and the times that you would simply focus on inhibiting theta, alpha, delta, or beta activity (without simultaneously reinforcing beta or SMR)? In terms of focusing for a period of time on inhibiting (rather than inhibiting plus reinforcing something else), for what types of problems or conditions would you use this strategy? Would you be inclined to use this for the entire neurofeedback session, or for only part of it? How long might you strictly focus on inhibiting certain activity before considering using another neurofeedback strategy?

RESPONSE: Margaret E. Ayers, MA, Neuropathways EEG Imaging, Inc., Beverly Hills, California. 
The human body maintains homeostasis. In the EEG, one can see a distribution of frequencies ranging up to $40 \mathrm{~Hz}$ with a dominant frequency present during a specific task. For example, in stage three or four of sleep, theta and delta waves are dominant. The brain maintains homeostasis by inhibitory chemo-architecture and neuronal mechanisms.

When the brain is injured, abnormal phasic spike and slow wave theta appears. The brain does not know how to eliminate the abnormal pattern to restore homeostasis, so it attempts to counteract the effects of slow theta waves by producing more beta activity. If we inhibit the theta activity, brain function improves, including memory, energy, concentration, and it becomes less sensitive to sound and light. When therapists increase beta in head injury, stroke, anoxia, and learning disabilities, the theta increases and makes the symptomatology worse. As mentioned above, when theta increases due to injury, beta also increases as a function of the brain's compensatory mechanism. The brain knows that it needs to operate faster than the slow activity produced when theta dominates, so it produces more fast activity to compensate.

From controlled research published in Science (1974), we know that normal individuals trained to produce theta activity during radar monitoring rapidly declined in performance. This study demonstrates that it is possible to upset homeostasis and produce iatrogenic effects. Theta frequency and rhythm is important in appropriate situations such as stage three and four sleep; but not appropriate in tasks requiring alertness, such as radar monitoring. The brain maintains this homeostasis of frequency, pattern, rhythm, and voltage in an inhibitory manner.

The exciting news is that if one looks at the primary EEG, not reconstructed, derived, and averaged, then it is possible to see what needs to be inhibited. The goal of EEG neurofeedback is to normalize the EEG so that the client feels better. My contention is that the brain does not know what to do until it first is told what not to do when abnormal activity is present. Using primary EEG data, focusing on inhibition of abnormal EEG activity, and normalizing the pattern results in permanent improvement of symptoms.

\section{REFERENCES}

Beatty, J., Greenberg, A., Deibler, W.P. \& O’Hanlon, J.F. (1974). Operant control of occipital theta rhythm affects performance in a radar monitoring task. Science (183), 871-873. 
RESPONSE: Marvin W. Sams, ND, Dallas, Texas. E-mail address: drmsams@aol.com

Before I begin Neurofeedback training, an IVA continuous performance test, a Quantitative EEG with cognitive challenges (eyes open, eyes closed, reading and listening to a text or story, and playing Tetris, a strategy video game) is done, and a Thatcher-Hudspeth database report run on both the eyes closed (normed) and Tetris (not normed) conditions. My clinical objective in this diagnostic process is to rule out structural pathology, determine the neurological inefficiencies, and to create a training strategy to remediate inappropriate electrophysiological findings. It is this information that dictates whether I do decrease training for Theta, Alpha, Delta, or Beta activity, as well as other types of training.

In my remedial process, I do a number of different types of training, including increase and decrease of inappropriate fast and slow wave frequencies using both referential and multi-electrode arrays in 3, 5, and 8 electrode site combinations (called LCC, or Linear Channel Combination, which is a feature of the Lexicor systems), and appropriate coherence and phase training. So, decrease training is just part of the mix.

Incidentally, when influencing slow wave activity, training is always done for magnitude decrease under task (as opposed to inhibiting slow waves while training fast frequencies), the results being measured with pre and post (no-audio, under task) baselines. Clinical experience has shown low Delta band activity $(0.5-2.0 \mathrm{~Hz})$ that is excessively high or increases or remains constant with cognitive task, to be the primary EEG feature in most of those with attentional issues, and it is quite common in mood disorders. For this reason, all new trainees, regardless of presenting diagnosis, receive decrease Delta training $(0.5-3 \mathrm{~Hz})$ under task (that is, while playing Tetris or another strategy-based video game), for twenty-five minute periods. This training is done early to maximize attention and provide early positive results. Each of the vertex electrodes is trained as individual sessions, up to six sessions total. When Delta decreases under task, even if it is first session, the training is considered complete and the next type of training on the strategy list is selected.

Research evidence shows that the brain uses Theta for such important tasks as memory, mood regulation, and spatial navigation. Also, 
those with frontal midline Theta (FmTheta-Fz electrode focus) at 6.2 $\mathrm{Hz}$ while under cognitive task are able to sustain attention for prolonged periods, and demonstrate low anxiety and neuroticism with an extroverted personality on psychological testing. Therefore, unless relative power elevations are present on the reference database, full band Theta is not trained for decrease. If full band Theta shows a statistical relative increase on the database, twenty-five minutes of decrease training is done referentially at an appropriate vertex electrode site. Increasing $5.5-8 \mathrm{~Hz}$ training at $\mathrm{Fz}$ (eyes open) is often very effective for increased FmTheta production, and is done routinely on each client-patient. Decreasing $3-5.5 \mathrm{~Hz}$ activity is also effective at the same electrode site, and is commonly done.

Low band Alpha at $8-10 \mathrm{~Hz}$ is decreased under task if elevated on the Tetris-based reference database printout. High band Alpha at $10-12 \mathrm{~Hz}$ is the second most important band for intellectual functioning (just behind $13 \mathrm{~Hz}$ [12-14 Hz]), so it is not usually trained for decrease. Technically, Beta is a special problem. EMG artifact is typically present in all EEG recordings, being especially prominent in the temporal and frontal areas. Even with almost four decades of clinical and research EEG experience, I am often hesitant to say, with absolute certainty, that a particular fast activity is beta or EMG. To further complicate the issue, temporalis muscle activity commonly spreads to one or both ear electrodes. In an ear reference recording, the artifactual fast activity may appear generalized (in all channels). The reference database will reflect this increased fast wave as a beta loading generally, or in particular channels, in all electrophysiological parameters. For these reasons, I do not train beta for decrease unless it is clear from the analog EEG that it is truly beta. For me, the only unequivocal evidence is "smooth" beta spindles, which usually occur in the fronto-temporal regions, and then, only if the client-patient is not taking drugs (which commonly increase beta activity). Rarely, I will do decrease beta training late in the training process if clinical issues remain and elevated beta deviations are present in the same electrode sites on the follow-up QEEG at session 20.

As a clinical suggestion, an effective Decrease Slow Wave training is to decrease Delta $(0.5-3 \mathrm{~Hz})$, Theta $(3-6 \mathrm{~Hz})$, and Alpha $(7-10 \mathrm{~Hz})$ for five minutes each, at the $\mathrm{Fz}, \mathrm{Cz}$, or $\mathrm{Pz}$ electrode site (as individual sessions). In all types of training, high-pitched tones are always used for audio. Reward is at the ninety-eight to one hundred percent level 
with "dips" in the audio tone every three to five seconds. Decrease slow wave training is only at the vertex electrodes, unless a slow wave focus is seen in the QEEG.

A final and most important note: Structural brain pathology (brain tumors and the like) are seen in the EEG as pronounced focal or diffuse slow waves (delta and/or theta activity). If such slow waves are present in the QEEG, the patient must be evaluated by a neurologist (or other medical doctor) before neurofeedback training is begun to avoid training a client-patient with intracranial pathology.

RESPONSE: M. Barry Sterman, PhD, Sterman-Kaiser Imaging Laboratory, Los Angeles, California.E-mail address: msterman@ucla.edu

Regarding the question of training slow frequencies down without accompanying reward for raising SMR or beta frequencies, I know of no published studies that have examined this. In my personal experience, I have found that it is essential to focus on reducing high voltage theta or slow alpha activity initially if this activity dominates the record. Most people can reduce these slower frequencies, at least for brief periods of time. But this alone will not succeed in permanently shifting the dominant frequency up, as desired. Reward for suppressing the slow frequencies must eventually be coupled with reward for enhancement of higher frequencies. Clients report being able to subjectively "feel" the effects of increasing higher frequencies, and can, therefore, learn some control over the associated state. With the slow frequencies they simply try to concentrate but have no other subjective cues on which to base learning-and mere concentration is both difficult to sustain and unlikely to improve functional regulation. These observations are also consistent with what we know about the underlying neurophysiology of EEG frequency patterns.

Training exclusively for the reduction of paroxysmal events, higher frequencies, or EMG activity, or for the enhancement of lower frequencies has been ineffective (Lubar et al., 1981; Sterman, 2000; Tozzo et al., 1988; Upton \& Longmire, 1975; Wyler et al., 1976). Additionally, Wyler et al., (1979), exploring the hypothesis that localized cortical activation was the operative factor in SMR training, attempted enhancement of higher frequency activity over variable sites of identified focal abnormality. Only twenty-seven percent of these patients showed meaningful seizure reductions. 
My answer would be that at least for the treatment of seizures, reward for SMR activity (which includes frequencies from $12-19 \mathrm{~Hz}$ recorded from central cortex) is essential for optimal therapeutic effects.

\section{REFERENCES}

Lubar, J. F., Shabsin, H. S., Natelson, S. E., Holder, G.S., Whitsett, S.F., Pamplin, W.E., \& Krulikowski, D.I. (1981). EEG operant conditioning in intractible epileptics. Archives of Neurology, 38, 700-704.

Sterman, M. B. (2000). Basic concepts and clinical findings in the treatment of seizure disorders with EEG operant conditioning. Clinical Electroencephalography, 31(1), 45-55.

Tozzo, C. A., Elfner, L. F., \& May Jr., J. G. (1988). EEG biofeedback and relaxation training in the control of epileptic seizures. International Journal of Psychophysiology, 6, 185-194.

Upton, A. R., \& Longmire, D. (1975). The effects of feedback on focal epileptic discharge in man: A preliminary report. Canadian Journal of Neurological Science, 2, 153-167.

Wyler, A. R., Lockard, J. S., \& Ward, A. A. (1976). Conditioned EEG desynchronization and seizure occurrence in patients. Electroencephalography \& Clinical Neurophysiology, 41(5), 501-512.

Wyler, A. R., Robbins, C. A., \& Dodrill, C. B. (1979). EEG operant conditioning for control of epilepsy. Epilepsia, 20, 279-286.

RESPONSE: Joel Lubar, PhD, University of Tennessee, Knoxville, Tennessee.E-mail address: jlubar@utk.edu

My response to the question about whether to inhibit theta or reinforce beta, SMR or other frequencies in working with attention deficit disorder is based on our experiences going back to 1975. We have developed a protocol, which is being used worldwide with considerable success. It is not the only possible protocol, but a good starting point. Based on both single channel and multichannel quantitative EEG analysis, we find that a majority of the individuals experiencing the inattentive type of ADD have either increased theta activity in terms of percent power and some times microvolts as well and/or decreased beta activity. More commonly in children approximately up to the age of about ten or eleven, the primary problem is excessive 4-8 $\mathrm{Hz}$ activity. In older children and adolescents, we see elevated activity in the $6-10 \mathrm{~Hz}$ range which I have designated theta-alpha or "thal- 
pha." These individuals sometimes show as well, decreased activity in the range anywhere from 16-20 Hz. The basic strategy then for the inattentive type is to set an initial inhibit level for the slow activity whether it be $4-8 \mathrm{hz}$ or $6-10 \mathrm{hz}$ with the percent time of inhibit of fifty percent for children, sixty percent for adolescents, and as high as seventy percent for older adolescents and adults. We set the reinforcement for beta activity at fifty percent for children, forty percent for adolescents and young adults, and thirty percent for older adults. For example, in a twenty-year-old we might set the instrumentation so that when they produce beta activity thirty percent of the time and inhibit the slow activity seventy percent of the time, in order to achieve the reward criteria. We also set an additional inhibit for EMG activity so that if the EMG activity occurs above threshold more than twenty percent of the time, it is inhibited. This essentially takes care of the large excursions due to head movements, body movements, gritting teeth, and generalized muscle activity. Once we have set a level of reward and inhibit, we do not change this during either a session or over sessions until a learning curve has been generated so that the conditions are essentially reversed. In other words, if the initial settings are sixty percent inhibit and forty percent reward, we will have the individual work until they have increased their reward level to sixty percent and their inhibit level has decreased to forty percent. At that point, we then make the criteria more difficult, essentially resetting them to the original level and then generate a new learning curve. Over the course of anywhere from thirty to fifty sessions, a person may generate two or three separate learning curves for these different criteria. The reason we do not switch criteria during a session or over sessions, is that it becomes confusing for the patient and difficult for them to track their progress. We use the graphs extensively to show the parents and the individual being trained their progress and we use the principles of learning theory in terms of generating learning curves. We do employ shaping in the sense of changing the criteria when they have met certain criterions as mentioned previously.

Sometimes an individual will be stuck on a plateau where they do not seem to advance. This is one of the cases where we will make the conditions somewhat easier so that we can try to reinitiate the learning curve if at all possible. This is one of the rare conditions in which we will actually change parameters either during a session or after a small number of sessions have occurred in which no progress is being made. 
I have tried autoscaling in the past to see if it works. The problem with it is that when the threshold automatically changes the criteria, patients become lazy and they stop working as hard so that the criteria become reset to easier and easier levels so that eventually the reinforcements are almost noncontingent or at least relatively meaningless.

If the individual is hyperactive as well as inattentive (which accounts for approximately fifty percent of our patient load), we start out with the inhibition of the slow activity whether it be theta or thalpha or low alpha and reward the sensorimotor rhythm instead with electrode placements over the motor strip either referential or bipolar depending upon initial measurements using both electrode montages. We use the same technique as we do for the theta-beta paradigm except that now we are training SMR. Once they have achieved a good learning curve with this procedure, we then switch to the theta-beta or thalpha-beta protocol with the electrode placements being shifted from the sensorimotor cortex to the midline. Our success rate over the years has been very high. Approximately eighty percent of the patients we work with show significant improvements in terms of continuous performance measurements and/or changes in psychometric measurements, school performance, and social adjustment as measured by different types of rating scales. As quantitative EEG elucidates more subtypes of ADD and associated co-morbidities, the protocols will be changed in terms of location of training and band passes, but the basic concepts of generating learning curves and using solid principles based on learning theory will still be applicable.

\section{MEDICAL JUSTIFICATION FOR NEUROFEEDBACK WITH ADD/ADHD}

QUESTION: What do I tell my patients who say there is no medical justification for neurofeedback for $\mathrm{ADD} / \mathrm{ADHD}$, and that medication is the only treatment proven effective?

RESPONSE: D. Corydon Hammond, PhD, Professor, Physical Medicine \& Rehabilitation, University of Utah School of Medicine. E-mail address: D.C.Hammond@m.cc.utah.edu

It should be explained that quantitative EEG research on ADD/ ADHD has verified that it involves brain abnormalities, with disturbed 
brainwave patterns that usually consist of excessive slow wave activity. Neuroimaging research using PET and SPECT scans provide further confirmation of the physiological basis of $\mathrm{ADD} / \mathrm{ADHD}$ problems. The brain abnormalities documented by this research provide medical justification for two primary approaches to treatment: medication and neurofeedback.

In ADD/ADHD, essentially the frontal lobe is "lazy" in focusing attention and in inhibiting motor activity (Niedermeyer \& Naidu, 1997). I explain that the front of the brain represents our executive control centers, which regulate attention, emotion, and behavior. In $\mathrm{ADD} / \mathrm{ADHD}$, there is almost always slower brainwave activity in the frontal areas of the brain. This physiological deficit in ADD/ADHD, I explain, makes it difficult to control attention. Therefore, persons with ADD/ADHD typically are easily distracted, have a short attention span, and have difficulty following directions and listening. When faster brainwaves are present, the inhibitory process associated with them serves to "filter" incoming sensory information so that the brain is not overwhelmed with stimulus input overload, allowing the individual to focus and concentrate. Likewise, persons with ADD/ADHD tend to lack inhibitory control over their emotions, making them more prone to tantrums, mood swings, and emotional outbursts. The slow brainwave activity also means that there is a deficiency in the brain's inhibitory process that puts the "brakes" on behavioral impulses, thereby making it difficult to regulate impulses and control one's own actions. Therefore, persons with ADHD have difficulty controlling their behavior, tending to be impulsive, fidgety, overly talkative, and hyperactive. Thus, these physiological deficits document that there absolutely is medical justification for neurofeedback to be used in remediation of the brain.

We can acknowledge that medication has been the primary treatment for ADD/ADHD and that we should be grateful that frequently medications do produce improvements during the times of day when the medication is being taken. We can note that although one study found that sixty-nine percent of children on Ritalin or other stimulants suffered from one or more side effects, the extent to which side effects are troublesome certainly varies. However, we can also point out that although medication can play a valuable role in assisting the person to manage ADD/ADHD, a massive recent review of literature found that medication did very little for twenty-five to forty percent of children 
with these problems. A thorough review of studies on stimulant use recently reported that stimulants provide "temporary improvement," but "on the other hand, changes that point toward longer-term improvement (e.g., in academic outcome, antisocial behavior, or arrest rate) were not found, and only small effects were observed on learning and achievement."

A report from the Council on Scientific Affairs of the American Medical Association (Goldman et al., 1998) concluded pharmacotherapy alone, while effective in short-term symptomatic improvement, "has not yet been shown to improve the long-term outcome for any domain of functioning (classroom behavior, learning, impulsivity, etc.)." The latest research by Chabot at NYU (Chabot \& Serfontein, 1996) found that stimulants produced behavioral improvement in only fifty-eight percent of children whose ADD is associated with a theta excess, while twenty-six percent were not helped, and sixteen percent actually had increased behavioral problems with stimulant treatment. In ADHD children with an excess of theta brainwaves, only thirtythree percent respond to stimulant medication, thirty-seven percent don't respond, and thirty percent get worse. In ADHD patients with excess alpha, only fifty percent respond to stimulants. All of this can be cited to patients and parents. However, it seems unethical to suggest to patients that medication is not helpful. It is often useful in treatment, and also while neurofeedback training is being conducted. However, we should discourage it from being seen or presented to the public or to professionals as the "one true light." We should emphasize that both neurofeedback and medication are viable treatment options, and medication may particularly be useful until neurofeedback training has been completed.

When patients have talked with sources that indicate that neurofeedback is "experimental," we can explain that there has been increasingly refined research that has been done since the 1970s. There are now many research studies and the long-term follow-ups suggest that in about eighty percent of cases neurofeedback substantially reduces the symptoms of ADD/ADHD. Furthermore, post-treatment intelligence testing commonly documents increases of ten to twenty IQ points. Interested patients may also be referred to a discussion of neurofeedback for $\mathrm{ADD} / \mathrm{ADHD}$ written for the general public that may be found on the web at Americasdoctor.com, in their ADD/ ADHD "community." 


\section{REFERENCES}

Amen, D. G., \& Carmichael, B. D. (1997). High-resolution brain SPECT imaging in AD/HD. Annals of Clinical Psychiatry, 9(2), 81-86.

Chabot, R. J., Merkin, H., Wood, L. M., Davenport, T. L., \& Serfontein, G. (1996). Sensitivity and specificity of QEEG in children with attention deficit or specific developmental learning disorders. Clinical Electroencephalography, 27(1), 26-34.

Chabot, R. J., \& Serfontein, G. (1996). Quantitative EEG profiles of children with attention deficit disorder. Biological Psychiatry, 40, 951-963.

Goldman, L.S., Genel, M., Bezman, R. J., \& Slanetz, P. J. (1998). Council Report: Diagnosis and treatment of attention-deficit/hyperactivity disorder in children and adolescents. Journal of the American Medical Association, 279(14), 1100-1107.

Linden, M., Habib, T., \& Radojevic, V. (1996). A controlled study of the effects of EEG biofeedback on cognition and behavior of children with attention deficit disorder and learning disabilities. Biofeedback \& Self-Regulation, 21(1), 35-49.

Lubar, J. F. (1995). Neurofeedback for the management of attention-deficit/hyperactivity disorders. Chapter in M. S. Schwartz (Ed.) Biofeedback: A Practitioner's Guide. (pp.493-522), New York: Guilford.

Lubar, J. F., Swartwood, M. O., Swartwood, J. N., \& O’Donnell, P. H. (1995). Evaluation of the effectiveness of EEG neurofeedback training for ADHD in a clinical setting as measured by changes in T.O.V.A., scores, behavioral ratings, and WISC-R performance. Biofeedback \& Self-Regulation, 20(1), 83-99.

Mann, C. A., Lubar, J. F., Zimmerman, A.W., Miller, B. A., \& Muenchen, R. A. (1992). Quantitative analysis of EEG in boys with attention deficit/hyperactivity disorder (ADHD). A controlled study with clinical implications. Pediatric Neurology, 8, 30-36.

Neidermeyer, E., \& Naidu, S.B. (1997). Attention-deficit hyperactivity disorder (ADHD) and frontal-motor cortex disconnection. Clinical Electroencephalography, 28, 130-136.

Othmer, S., Othmer, S. F., \& Kaiser, D. A. (1999). EEG biofeedback: Training for $\mathrm{AD} / \mathrm{HD}$ and related disruptive behavior disorders. Chapter in J. A. Incorvaia, B. F. Mark-Goldstein, \& D. Tessmer (Eds.), Understanding, Diagnosing, \& Treating AD/HD in Children and Adolescents. (pp. 235-297). New York: Aronson.

Rossiter, T. R., \& La Vaque, T. J. (1995). A comparison of EEG biofeedback and psychostimulants in treating attention deficit/hyperactivity disorders. Journal of Neurotherapy, 1(1), 48-59.

Tansey, M. A. (1990). Righting the rhythms of reason: EEG biofeedback training as a therapeutic modality in a clinical office setting. Medical Psychotherapy, 3, 57-68.

Zametkin, A. J., Nordahl, T. E., Gross, M. et al. (1990). Cerebral glucose metabolism in adults with hyperactivity of childhood onset. New England Journal of Medicine, 323(2), 1361-1366. 